\title{
DEEPSEN: a convolutional neural network based method for super-enhancer prediction
}

\author{
Hongda Bu', Jiaqi Hao', Yanglan Gan³, Shuigeng Zhou² and Jihong Guan ${ }^{1 *}$ \\ From 14th International Symposium on Bioinformatics Research and Applications (ISBRA'18) \\ Beijing, China. 8-11 June 2018
}

\begin{abstract}
Background: Super-enhancers (SEs) are clusters of transcriptional active enhancers, which dictate the expression of genes defining cell identity and play an important role in the development and progression of tumors and other diseases. Many key cancer oncogenes are driven by super-enhancers, and the mutations associated with common diseases such as Alzheimer's disease are significantly enriched with super-enhancers. Super-enhancers have shown great potential for the identification of key oncogenes and the discovery of disease-associated mutational sites.

Results: In this paper, we propose a new computational method called DEEPSEN for predicting super-enhancers based on convolutional neural network. The proposed method integrates 36 kinds of features. Compared with existing approaches, our method performs better and can be used for genome-wide prediction of super-enhancers. Besides, we screen important features for predicting super-enhancers.
\end{abstract}

Conclusion: Convolutional neural network is effective in boosting the performance of super-enhancer prediction.

Keywords: Super-enhancer prediction, Deep learning, Convolutional neural network

\section{Background}

Numerous transcriptional factors combine with enhancers to regulate gene expression through recruiting transcriptional coactivator and RNA polymerase to target gene [1]. The term 'enhancer' was first introduced to describe the effects of SV40 DNA on the ectopic expression of a cloned rabbit $\beta$ globin gene. The SV40 DNA elements activated transcription at a distance and independently of their orientation concerning the target gene [2]. Enhancer activation often coincides with DNase I hypersensitivity of these regions and with specific posttranslational modifications of adjacent nucleosomes [3]. Direct interaction or looping between enhancers and the promoters of target genes has been observed and might be critical to enhancer function $[4,5]$. Recently, advances

*Correspondence: jhguan@tongji.edu.cn

${ }^{1}$ Department of Computer Science and Technology, Tongji University, 4800 Cao'an Road, Shanghai 201804, China

Full list of author information is available at the end of the article in DNA sequencing technology, such as Chromatin Immunoprecipitation sequencing(ChIP-seq) and DNase I hypersensitivity sites sequencing(DNase-seq) have enabled the discovery of putative mammalian enhancers on a genome-wide scale [6-10].

The concept of super-enhancers was proposed by Richard A.Young based on the research on enhancers, which is described as a class of regulatory regions with unusually strong enrichment for the binding of transcriptional coactivators, specifically Mediator (Med1) $[11,12]$. In mouse embryonic stem cells (mESCs), superenhancers were defined in the following way [12]: 1) Sites bound by all three master regulators, Oct4, Sox 2 and Nanog, according to ChIP-seq, were considered enhancers; 2) Enhancers within $12.5 \mathrm{~kb}$ of each other were stitched to define a single entity spanning a genomic region; 3) The stitched enhancer entities and the remaining individual enhancers (those without a neighboring enhancer within $12.5 \mathrm{~kb}$ ) were then ranked by the total background-normalized level of the Med1 signal within 
the genomic region. A small proportion (less than 3\%) of these enhancer regions contained Med1 levels above a cutoff was designated as super-enhancers. The remaining enhancer regions were considered 'normal' enhancers. Super-enhancers tend to span large genomic regions, whose median size generally an order of magnitude larger than that of normal enhancers (in mESCs, 8667 bp versus 703 bp) [11-13]. Relative to Med1, a number of factors generally associated with enhancer activity show enrichment at super-enhancers relative to normal enhancers. These factors include RNA polymerase II (Pol II), RNA from transcribed enhancer loci (eRNA), the histone acetyltransferases $\mathrm{p} 300$ and CBP, chromatin factors such as cohesin, the histone modifications H3K27ac, H3K4me2 and H3K4me1, and increased chromatin accessibility as measured by DNase-seq. Because of these crosscorrelations, super-enhancers might be identified by many of these features [11].

Since super-enhancers influence various biological processes, the identification of super-enhancers becomes an urgent research issue. BRD4, a member of the BET protein family, was used to distinguish super-enhancers from typical enhancers as it is highly correlated with MED1 [13]. H3K27ac was extensively used to create a catalog of super-enhancers across 86 different human cell-types and tissues due to its availability [11]. Other studies used the coactivator protein P300 to define superenhancers [14, 15] However, the knowledge about these factors' ability to define a set of super-enhancers in a particular cell-type and their relative and combinatorial importance remains limited. Master transcriptional factors that might form super-enhancers domains are largely unknown for most cell-types, while performing ChIPseq for the Mediator complex is difficult and costly. However, there are no predictive models that integrate various types of data to predict super-enhancers and their constituents (enhancers within super-enhancer). Besides, to what degree these features influence on superenhancers remains unknown.

Predicting super-enhancers based on machine learning remains nearly blank in the literature. The only work was done by Khan and Zhang [16]. They used six different machine learning models, including Random Forest, linear SVM, KNN, AdaBoost, Naive Bayes and Decision Tree. Chromatin, transcription factors and sequence-specific features were used to train these models individually, which were evaluated by 10 -fold crossvalidation. With the rise of deep learning (DL) techniques, many researchers applied state-of-art DL methods to bioinformatics problems. In DEEPBIND [17], Alipanahi et al. described the use of a deep learning strategy to calculate protein-nucleic acid interactions from diverse experimental data sets. Their results showed DL's applicability in bioinformatics and improved prediction power over traditional methods. Besides, Zhou et al. developed a deep-learning based algorithmic framework, named DeepSEA, which learns a regulatory sequence code from large-scale chromatin-profiling data in order to predict the noncoding variants effects [18].

In this work, we proposed a novel approach to solving the problem of super-enhancer prediction based on convolutional neural networks (CNNs). This method is called DEEPSEN. We constructed different structures of $\mathrm{CNN}$ to discover which kind of structure is more appropriate for the problem. For each network structure, we did fine-tuning to find out the best parameter set and to avoid overfitting. Furthermore, we did feature ranking and found out the significance of features for super-enhancers prediction. Our experimental results demonstrate that DEEPSEN outperforms the existing super-enhancer prediction model.

\section{Methods}

\section{Datasets}

Similar to Aziz Khan [16], we obtained 32 publicly available ChIP-seq and DNase-seq datasets of mouse embryonic stem cells (mESC) from Gene Expression Ominibus (GEO). These data cover four histone modifications (H3K27ac, H3K4me1, H3K4me3 and H3K9me3), DNA hypersensitive site (DNaseI), RNA polymeraseII (Pol II), transcriptional co-activating proteins (p300 and CBP), P-TFEb subunit (Cdk9), sub-units of Mediator complex (Med1, Med12 and Cdk8), chromatin regulators (Brg1, Brd4 and Chd7), Cohesin (Smc1 and Nipbl), subunits of Lsd1-NuRD complex (Lsd1 and Mi2b) and 11 transcription factors (Oct4, Sox2, Nanog, Esrrb, Klf4, Tcfcp2l1, Prdm14, Nr5a2, Smad3, Stat3 and Tcf3). Table 1 shows the datasets used in this paper.

We used MED1 signal to define super-enhancers as described in ROSE [12]. We selected transcriptional enriched regions as the training samples. Thus, we obtained 11100 samples with 36 kinds of features. Among them, 1119 are positive samples and 9981 are negative ones.

\section{Pipeline of the dEEPSEN method}

Based on convolutional neural network (CNN), we proposed a novel approach named DEEPSEN to predict super enhancers on genome scale. Fig. 1 illustrates the pipeline of the DEEPSEN method. It consists of three major steps:

1 Data preprocessing and feature calculation. 36 kinds of features were used to represent super-enhancers, including DNA sequence compositional features, histone modifications, transcriptional factors, RNA polymeraseII, hypersensitive site, co-activators, chromatin regulators, cohesion, mediator complex, mediator complex, and Lsd1-NuRD complex. 
Table 1 Datasets used in this paper

\begin{tabular}{lll}
\hline Data Type & Data Name & GEO ID \\
\hline Transcription factors & Oct4, Sox2, Nanog, Esrrb, Klf4, Smad3, & GSE44286, GSM288355, GSM288354, GSM623989, \\
Tcfcp2l1, Prdm14, Stat3, Tcf3, Nr5a2 & GSM53954 \\
Mediator complex & MED1 & GSM560348,GSM560345 \\
Histone modifications & H3K27ac, H3K4me1, H3K4me3, H3K9me3 & GSM594579, GSM281695, GSM307149, GSM18371 \\
RNA polymerase & RNA Pol & GSM318444 \\
Hypersensitive site & DNasel & GSM1014154 \\
Co-activators & p300, CBP & GSM918750,GSM1246866 \\
Chromatin regulators & Brg1, Brd4, Chd7 & GSM896923, GSM937540, GSM558674 \\
Cohesion & Nipbl, Smc1 & GSM560350,GSM560342 \\
Mediator complex & MED12 & GSM560348,GSM560345 \\
Lsd1-NuRD complex & Lsd1, Mi2b & GSM687282,GSM687284 \\
\hline
\end{tabular}

2 Constructing and training DEEPSEN. First, we built three models with different numbers of convolutional layers. Then, we trained each model using the back propagation (BP) algorithm [19] and stochastic gradient descent optimization algorithm. Furthermore, we did parameter tuning and validated each model using 5-fold cross-validation.

3 Feature ranking. We evaluated each feature's contribution to the identification of super-enhancers.

In what follows, we elaborate the process of superenhancer prediction step by step.

\section{Data preprocessing and feature selection}

Firstly, we aligned the original ChIP-seq reads to mouse genome-build $\mathrm{mm} 9$ with bowtie 0.12.9 [20]. As a result, we got the start and end positions of each read. Secondly, with these positions and the help of bamtoGFF, we calculated the read densities of samples, including super-enhancers and normal enhancers, and normalized these densities. Thirdly, we evaluated the binding affinity scores of all the samples with DNA binding motif information. Finally, we combined the calculated read densities and the binding affinity scores to get the final training data.

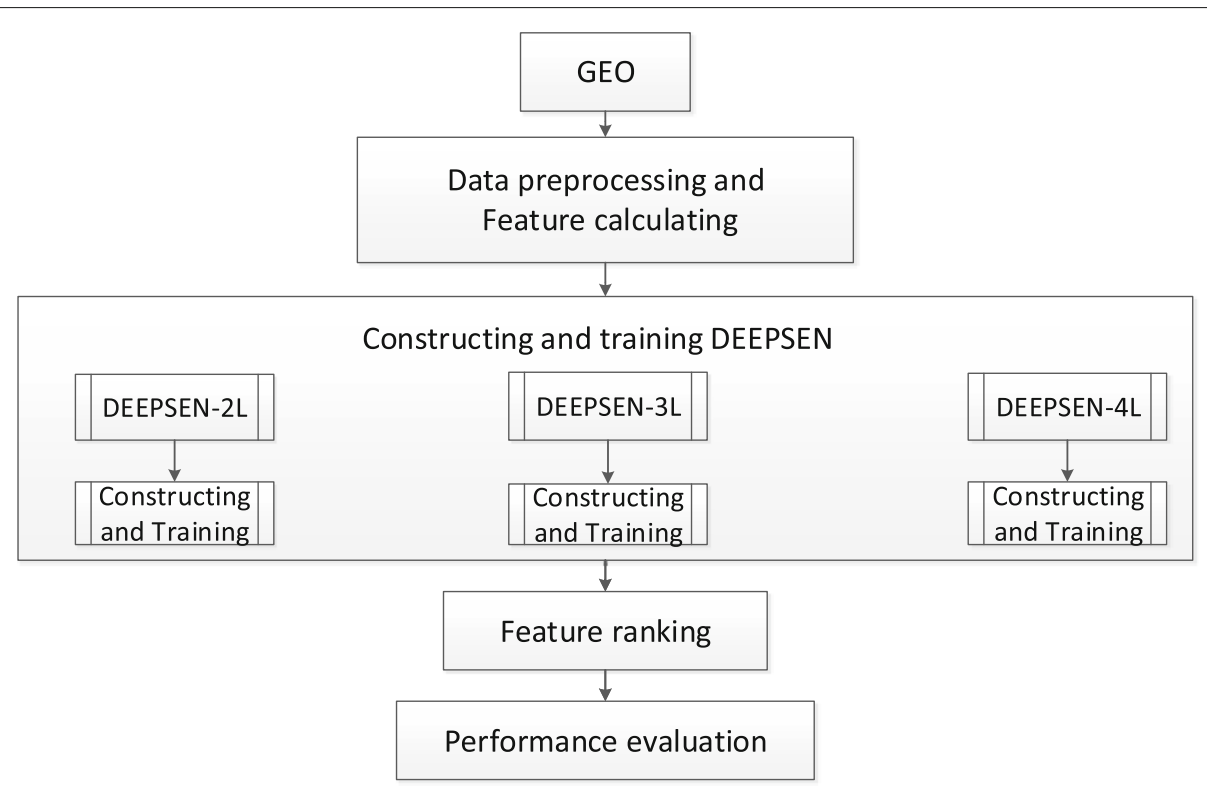

Fig. 1 The pipeline of DEEPSEN. The data we used were from GEO. Firstly, we do data preprocessing and feature calculation. Secondly, we construct three models with different numbers of convolutional layers and train them. Thirdly, we evaluate Pearson correlation coefficient to rank the features for predicting super-enhancers. Finally, we do performance evaluation and analysis 


\section{Constructing and training dEEPSEN The structure of dEEPSEN}

Figure 2 shows the architecture of a DEEPSEN classifier, which consists of the input layer (the 1st convolutional layer, including max-pooling), the 2nd convolutional layer (including max-pooling), ..., the fully connected layers, and the output layer.

The convolutional layer contains two steps: convolution step and pooling step. The convolution step uses multiple convolutional kernels to do convolution operation on the input data. A max-pooling operation often follows a convolution step to output a local maximal value of the respective convolutional outputs. The convolution operation learns to recognize relevant patterns of the input. The function of max-pooling is to reduce parameters to abstract the features learned in the proceeding layers. An activation function is usually used after each layer, which is nonlinear to guarantee the nonlinearity of the whole model. Here, we used the rectified linear unit(ReLU) function:

$$
\operatorname{ReLU}(x)=\max (0, x)
$$

The subsequent convolutional layers capture the relationships of the features extracted from the proceeding layers to obtain high-level features. Finally, the fully connected layer with dropout transforms the input into probability distribution through the softmax function:

$$
f_{i}(z)=\frac{\mathrm{e}^{z_{i}}}{\sum_{j} \mathrm{e}^{z_{j}}}
$$

The parameter details of the architecture are described in Table 1 . We take the model consisting of 2 convolutional layers as the example. The input layer is a $\mathrm{N} \times 36 \times 1$ matrix, where $N$ is the number of samples that is set to 11100 in our experiments. The first convolutional layer contains 32 kernels of shape $3 \times 1$ with the stride of 1 using the same padding so that the size does not change during convolution operation with. The output of the first layer includes 32 feature maps of size $36 \times 1$. Next is the first pooling layer of size $3 \times 1$, which means that we remain only the maximum value among every three values to reduce the dimensions and make the model robust. The second convolutional layer has 64 kernels, each of which is $3 \times 1 \times 32$, and its output includes 64 feature maps of size $12 \times 1$. The 2 nd pooling layer uses $3 \times 1$ max-pooling, and its output contains 64 feature maps of size $4 \times 1$, that is, $64 * 4=256$ nodes. Following is the fully connected layer with 256 input nodes and 64 output nodes. We used dropout method [21] in the fully connected layer to delete some nodes randomly for controlling over-fitting. The detailed structure of DEEPSEN that contains two convolutional layers is presented in Table 2. Besides the DEEPSEN with two convolutional layers, we also constructed DEEPSEN predictors with three convolutional layers and four convolutional layers. The details are presented in Tables 3 and 4, respectively.

The major difference between the CNN based models and previous models lies in that $\mathrm{CNN}$ can learn to recognize relevant patterns of input by updating the network during training. Therefore, the advantage of $\mathrm{CNN}$ based models is the ability to learn complicated features from large-scale datasets in an adaptive manner.

\section{The training of $d E E P S E N$}

We used the cross entropy loss function, which is as follows:

$$
J(\theta)=-\frac{1}{m} \sum_{i=1}^{m} y^{i} \log \left(h_{\theta}\left(x^{i}\right)\right)+\left(1-y^{i}\right) \log \left(1-h_{\theta}\left(x^{i}\right)\right)
$$

where $\theta$ is the parameter set, $\mathrm{m}$ is the amount of samples, $\mathrm{y}^{i}$ is the label of $\mathrm{x}^{i}, \mathrm{~h}_{\theta}\left(\mathrm{x}^{i}\right)$ is the predicted label of $\mathrm{x}^{i}$. Parameters were randomly initialized. The data was processed from the input layer to the output layer, and back propagation [19] and stochastic gradient descent

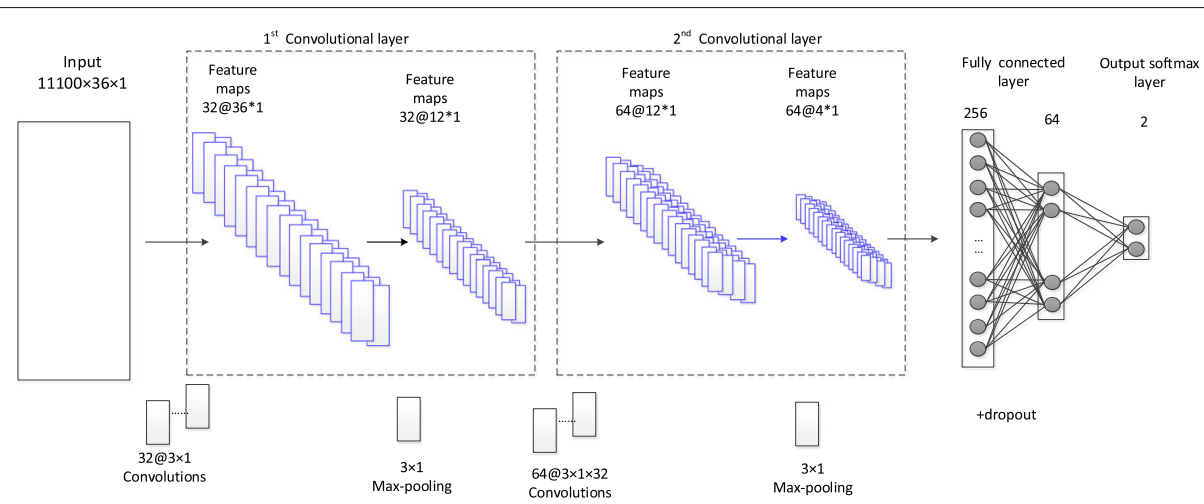

Fig. 2 The architecture of DEEPSEN-2L. DEEPSEN-2L consists of the input layer, the 1st convolutional layer (including the 1st max-Pooling), the 2nd convolutional layer (including the 2nd max-Pooling), fully connected layer (including dropout), and output softmax layer 
Table 2 The structure of DEEPSEN-2L

\begin{tabular}{lll}
\hline Layer & Size & Output Shape \\
\hline Input & & $36 \times 1$ \\
Convo1 & $32 \times 3 \times 1$ & $32 \times 36 \times 1$ \\
Pool1 & $1 \times 3$ & $32 \times 12 \times 1$ \\
Convo2 & $64 \times 3 \times 1 \times 32$ & $64 \times 12 \times 1$ \\
Pool2 & $1 \times 3$ & $64 \times 4 \times 1$ \\
Full-connected & 256 & 64 \\
softmax & 64 & 2 \\
\hline
\end{tabular}

algorithms were used to update the network parameters to minimize the cost function. Each epoch contains forward propagation, loss calculation, back propagation and parameter refreshing. The detailed training steps are as follows:

1 Initializing the parameters randomly.

2 Feeding the training data to the input layer.

3 Doing convolution operation and max-pooling operation in each conventional layer

4 Using the output of the last convolutional layer as the input of fully connected layer to obtain the result of the output layer

5 Evaluating the cost function and doing Adam optimization [22] using the BP algorithm [19] to refresh the parameters

6 Repeating step 2 to step 5 (one epoch) to recalculate the cost function until the desirable number of iterations is reached.

\section{Feature ranking}

In our models, we integrated 36 different features to predict super enhancers, including H3K27ac, H3K4me1, H3K4me3, H3K9me3, Brd4, Cdk8, Cdk9, Med12, p300, CBP, Pol2, Lsd1, Brg1, Smc1, Nipbl, Mi2b, CHD7, HDAC2, HDAC, DNaseI, 4-Oct, Sox2, Nanog, Smad3, Stat3, Tcf3, Esrrb, Klf4, Prdm14, Tcfcp2I1, Nr5a2, AT

Table 3 The structure of DEEPSEN-3L

\begin{tabular}{lll}
\hline Layer & Size & Output Shape \\
\hline Input & & $36 \times 1$ \\
Convo1 & $32 \times 3 \times 1$ & $32 \times 36 \times 1$ \\
Pool1 & $1 \times 3$ & $32 \times 12 \times 1$ \\
Convo2 & $64 \times 3 \times 1 \times 32$ & $64 \times 12 \times 1$ \\
Pool2 & $1 \times 3$ & $64 \times 4 \times 1$ \\
Convo3 & $128 \times 3 \times 1 \times 64$ & $128 \times 4 \times 1$ \\
Pool3 & $1 \times 2$ & $128 \times 2 \times 1$ \\
Full-connected & 256 & 64 \\
softmax & 64 & 2 \\
\hline
\end{tabular}

Table 4 The structure of DEEPSEN-4L

\begin{tabular}{lll}
\hline Layer & Size & Output Shape \\
\hline Input & & $36 \times 1$ \\
Convo1 & $32 \times 3 \times 1$ & $32 \times 36 \times 1$ \\
Pool1 & $1 \times 3$ & $32 \times 12 \times 1$ \\
Convo2 & $64 \times 3 \times 1 \times 32$ & $64 \times 12 \times 1$ \\
Pool2 & $1 \times 3$ & $64 \times 4 \times 1$ \\
Convo3 & $128 \times 3 \times 1 \times 64$ & $128 \times 4 \times 1$ \\
Pool3 & $1 \times 2$ & $128 \times 2 \times 1$ \\
Convo4 & $256 \times 3 \times 1 \times 128$ & $256 \times 2 \times 1$ \\
Pool4 & $1 \times 2$ & $256 \times 1 \times 1$ \\
Full-connected & 256 & 64 \\
softmax & 64 & 2 \\
\hline
\end{tabular}

content, GC content, phastCons, phastConsP, re- peat fraction. To measure the predictive power of each feature, we computed the Pearson correlation coefficient between each feature vector and the output label vector of all test samples. Then, we ranked these features based on the calculated Pearson correlation coefficient.

\section{Results and discussion}

\section{Parameter tuning}

DEEPSEN was implemented on tensorflow [23] with python. To investigate the impact of the number of convolutional layers on prediction performance, we constructed three models with different layers of convolutional neural networks, concretely, two, three and four convolutional layers. For simplification, these models are denoted as DEEPSEN-2L, DEEPSEN-3L and DEEPSEN$4 \mathrm{~L}$, respectively.

For each model, although most parameters were tuned automatically in the training process of the convolutional neural networks, there are still some hyper-parameters to be determined. Here, the Adam optimization method [22] was applied. We used grid search to tune the hyper-parameters, including learning rate, the number of epoches and the number of layers. Based on a number of preliminary experiments, we limit the parameters in the following ranges: the number of layers $L$ : 2-4 (with stride 1 ); the number of epoches $e$ : 50-150 (with stride 10); learning rate $\alpha: 10^{-5}, 5 \times 10^{-5}, 10^{-4}, 5 \times 10^{-4}, 10^{-3}, 5 \times 10^{-3}$, $10^{-2}, 5 \times 10^{-2}$.

We used accuracy as evaluation metric to tune parameters. The results are shown in Fig. 3. For DEEPSEN-2L, when $\alpha$ is set between 0.00005 and 0.0001 , it achieves better prediction accuracy. Generally, the accuracy increases with the number of epoches (for the number of epoches $\leq 140$ ). We did not choose too large numbers of epoches for the reason of training efficiency. When $\alpha$ is set to between 0.01 to 0.05 , the accuracy is fixed at 0.9 


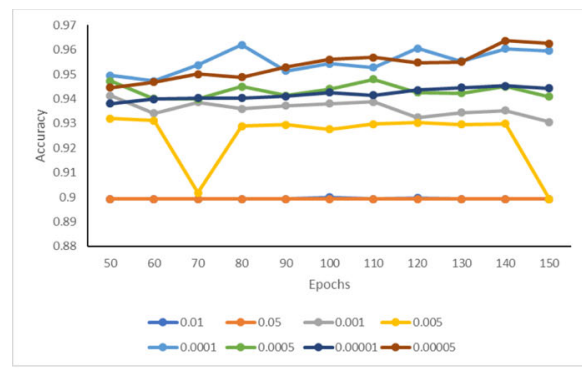

(a)

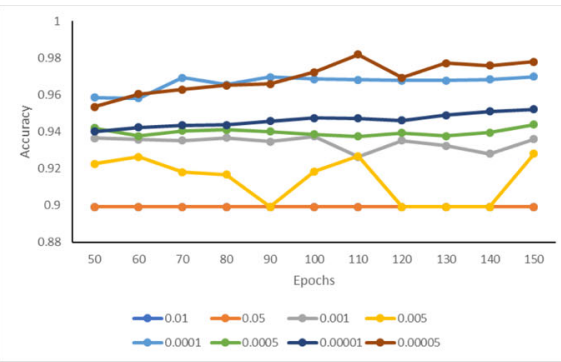

(b)

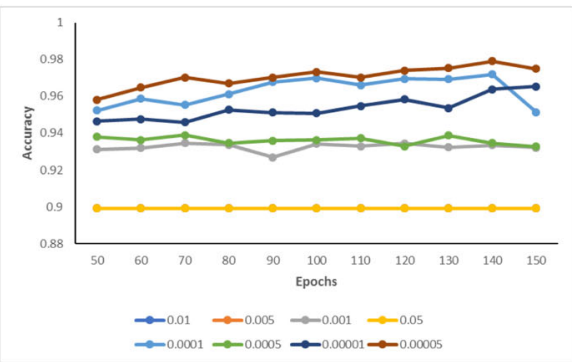

(c)

Fig. 3 Accuracy results under different parameter sets. a Accuracy vs. epochs for different $\alpha$ (DEEPSEN-2L); b Accuracy vs. epochs for different $\alpha$ (DEEPSEN-3L); c Accuracy vs. epochs for different? (DEEPSEN-4L)

because $\alpha$ is so large that gradient descent algorithm can not perform well, and DEEPSEN-2L predicts all samples as negatives (note that the ratio of negatives over positives is 9). DEEPSEN-3L and DEEPSEN-4L show similar patterns on parameters tuning. Overall, the optimized learning rate is between $5^{*} 10^{-4}$ and $10^{-4}$, the optimized number of epoches is between 140-150. With such parameter setting, DEEPSEN-4L achieves a better overall performance. Thus, we chose DEEPSEN-4L as the final model to predict super-enhancers. In what

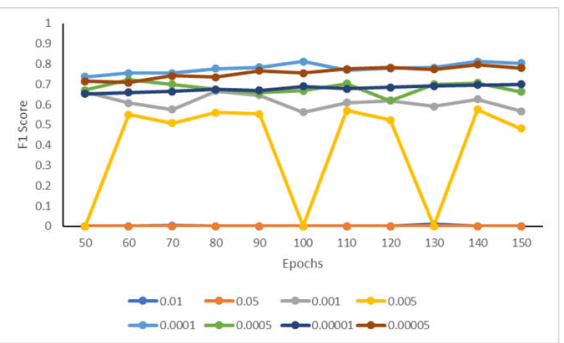

(a)

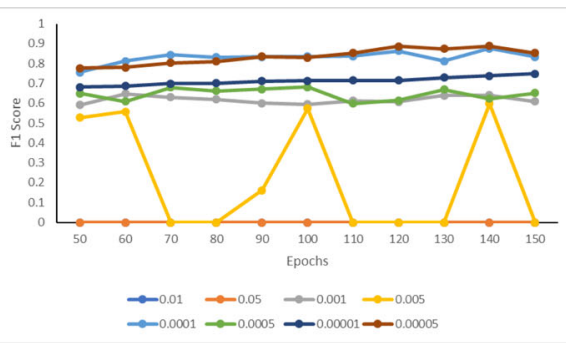

(b)

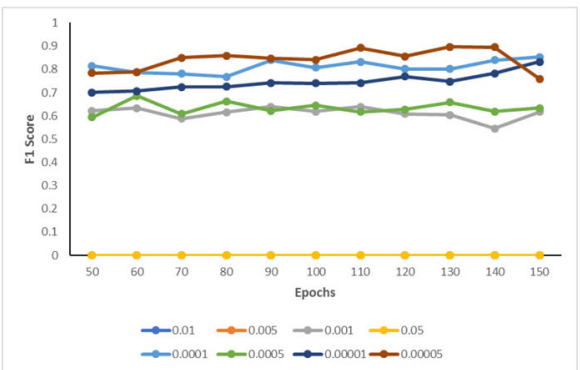

(c)

Fig. $4 \mathrm{~F} 1$ results under different parameter sets; a F1 vs. epochs for different $\alpha$ (DEEPSEN-2L). b F1 vs. epochs for different $\alpha$ (DEEPSEN-3L). c F1 vs. epochs for different $\alpha$ (DEEPSEN-4L) 
Table 5 Performance comparison with the state-of-the-art method

\begin{tabular}{lllll}
\hline Method & Precision & Recall & F1-score & AUC \\
\hline Improse & 0.88 & 0.81 & 0.84 & 0.97 \\
DEEPSEN & 0.92 & 0.88 & 0.90 & 0.97 \\
\hline
\end{tabular}

follows, we compare our three models with existing methods in terms of evaluation metrics precision, recall, F1 and $A U C$. The definitions of theses evaluation metrics is as follows. In classification task, TP denotes the true positives, FP denotes the false positives, TN denotes the true negatives and FN denotes the false negatives. ROC(Receiver Operating Characteristic) curve describe the relation between FP rate and TP rate, AUC is the area under curve.

$$
\begin{aligned}
& \text { Precision }=\frac{T P}{T P+F P} \\
& \text { Recall }=\frac{T P}{T P+F N} \\
& F 1=\frac{2 * \text { Precision } * \text { Recall }}{\text { Precision }+ \text { Recall }}
\end{aligned}
$$

\section{Performance evaluation}

The $F 1$ values of our three models under different hyperparameter settings are shown in Fig. 4. For DEEPSEN-2L, the best performance is achieved with $\alpha=0.0001$ and the number of epoches being 140. For DEEPSEN-3L, the best performance is obtained when $\alpha=0.00005$ and the number of epoches is 140 . As for DEEPSEN-4L, the best performance comes from $\alpha=0.00005$ and the number of epoches being 130. So we can see that all the three models of DEEPSEN achieve the best $F 1$ when $\alpha$ is between 0.00005 and 0.0001 , and the number of epoches is between 130 and 140. This observation is also noticed on accuracy.

The performance results of DEEPSEN with different structures are given in Table 5, where the performance results of improse [16] are listed for comparison. We can see that DEEPSEN-3L and DEEPSEN-4L perform better than improse in terms of precision, recall and F1. It demonstrates that the proposed DEEPSEN method outperforms the stat-of-the-art method improse. Figure 5 shows the performance comparison between our models and improse, and Fig. 6 shows the best AUC of DEEPSEN-4L when $\alpha=0.00005$ and the number of epoches is 110 .

\section{Performance comparison among different features}

The results of the first six correlated features are presented in Table 6. The Pearson correlation coefficient indicates the contribution of each feature to prediction performance. For our method, the feature ranking according to Pearson correlation coefficient is: Med12, cdk8,

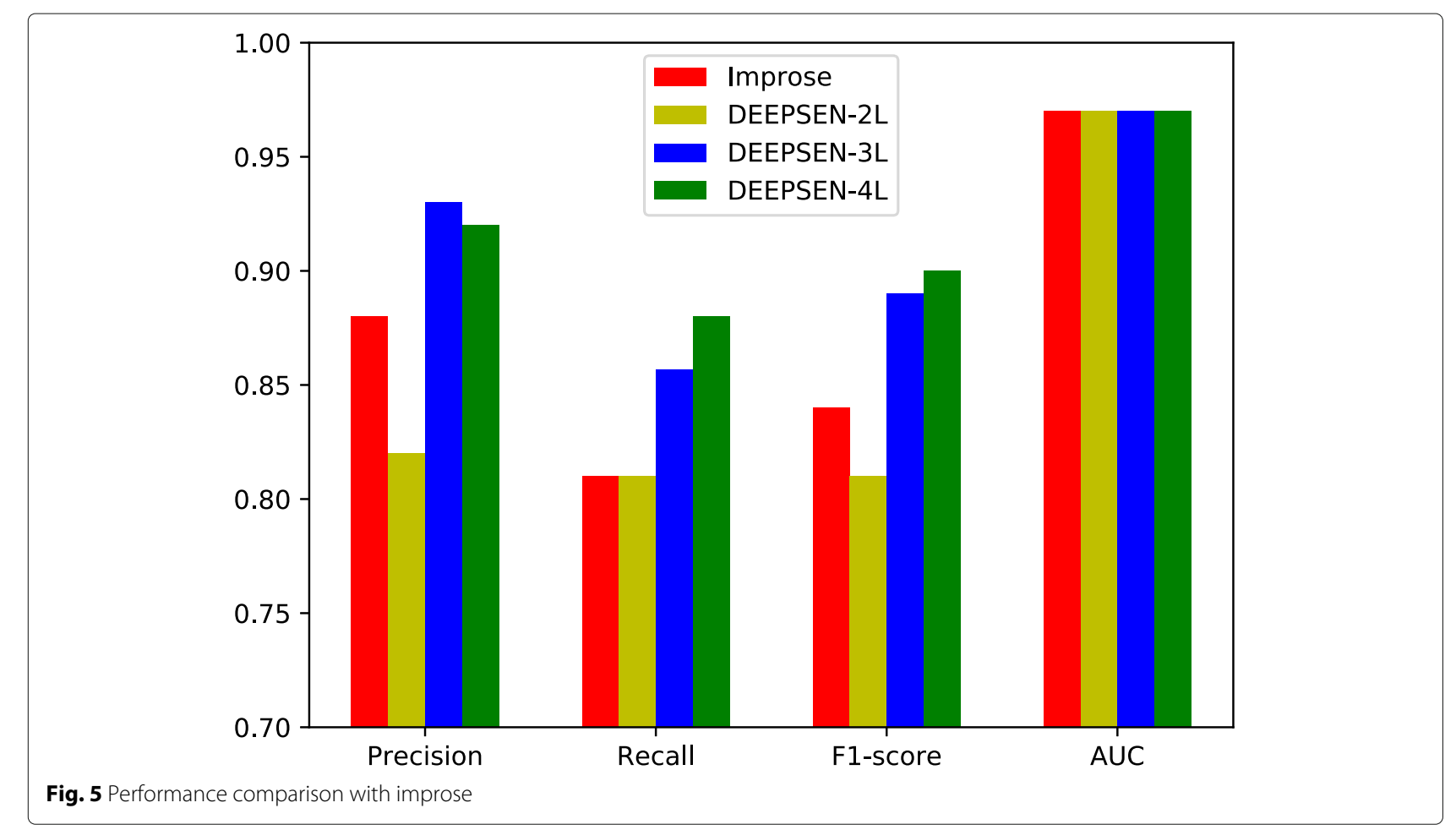




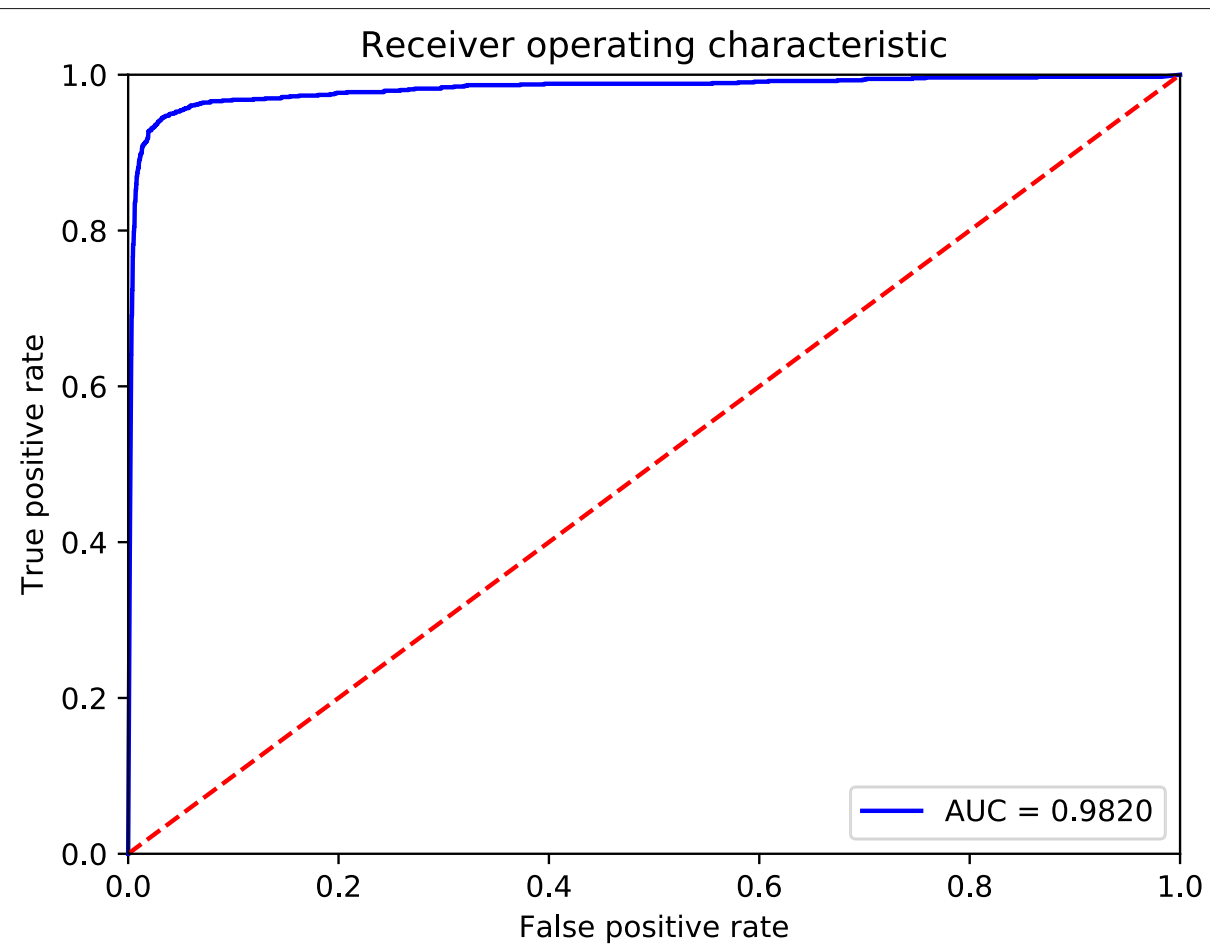

Fig. 6 The best ROC curve of DEEPSEN

Brd4, Cdk9, P300, H3K27ac, which is roughly similar to the findings of improse. The ranking given by improse is: Brd4, H3K27ac, Cdk8, Cdk9, Med12 and p300.

\section{Conclusion}

In this paper, we proposed DEEPSEN, a new superenhancer prediction method based on convolutional neural networks (CNNs). The data from GEO were used to train and test the proposed method. 36 kinds of features, including DNA sequence, histone modifications and TF bindings were integrated to train three models with 2, 3 and 4 convolutional layers. DEEPSEN uses a three-step scheme to construct and train CNN based classifiers. The first step is data preprocesing and feature calculation. The second step is to construct and train DEEPSEN. The third step is feature ranking. Our experimental results show that DEEPSEN outperforms the existing methods. DEEPSEN can be used with high-throughput experimental techniques to improve the accuracy of super-enhancer prediction.

Table 6 The results of feature ranking

\begin{tabular}{lllllll}
\hline Features & Med12 & Cdk8 & Brd4 & Cdk9 & p300 & H3K27ac \\
Correlation & 0.746 & 0.731 & 0.684 & 0.643 & 0.618 & 0.605 \\
\hline
\end{tabular}

\section{Abbreviations}

BP:Back-propagation; ChIP-seq:Chromatin Immunoprecipitation sequencing; CNNs:Convolutional neural networks; DL:Deep learning; DNase-seq:DNase I hypersensitive sites sequencing; eRNA:Enhancer RNA; GEO:Gene Expression Ominibus; KNN:K-nearest neighbors; mESCs:Mouse embryonic stem cells; SEs:Super-enhancers; SVM: Support vector machine

\section{Acknowledgements}

Not applicable.

\section{About this supplement}

This article has been published as part of BMC Bioinformatics, Volume 20 Supplement 15, 2019: Selected articles from the 14th International Symposium on Bioinformatics Research and Applications (ISBRA-18): bioinformatics. The full contents of the supplement are available at https://bmcbioinformatics. biomedcentral.com/articles/supplements/volume-20-supplement-15.

\section{Authors' contributions}

$\mathrm{JH}$ and SG designed the research and revised the manuscript. HD and JQ developed the method, carried out experiments, and drafted the manuscript. $Y L$ revised the manuscript. All authors have read and approved the final manuscript.

\section{Funding}

This work was funded by the National Natural Science Foundation of China (NSFC) (grant No. 61772367), which supported the collection, analysis and interpretation of data, the National Key Research and Development Program of China (grant No. 2016YFC0901704), which supported the publication costs, and the Shanghai Natural Science Foundation (grant No. 17ZR1400200), which supported the hardware and software device.

\section{Availability of data and materials}

The data and materials are available at https://github.com/1991Troy/DEEPSEN

\section{Ethics approval and consent to participate}

Not applicable. 


\section{Consent for publication}

Not applicable.

\section{Competing interests}

The authors declare that they have no competing interests.

\section{Author details}

${ }^{1}$ Department of Computer Science and Technology, Tongji University, 4800 Cao'an Road, Shanghai 201804, China. ${ }^{2}$ Shanghai Key Lab of Intelligent Information Processing, and School of Computer Science, Fudan University, 220 Handan Road, Shanghai 200433, China. ${ }^{3}$ School of Computer Science and Technology, Donghua University, 2999 North Renmin Road, Shanghai 201620, China.

Received: 2 October 2019 Accepted: 29 October 2019 Published: 24 December 2019

\section{References}

1. Pott S, Lieb JD (2015) What are super-enhancers?. Nat Genet 47(1):8-12

2. Banerji J, Rusconi S, Schaffner W (1981) Expression of a beta-globin gene is enhanced by remote sv40 dna sequences. Cell 27(2 Pt 1):299

3. Shlyueva D, Stampfel G, Stark A. (2014) Transcriptional enhancers: from properties to genome-wide predictions. Nat Rev Genet 15(4):272

4. Deng W, Lee J, Wang H, Miller J, Reik A, Gregory PD, Dean A, Blobel GA (2012) Controlling long range genomic interactions at a native locus by targeted tethering of a looping factor. Cell 149(6):1233-44

5. Tolhuis B., Palstra R. J., Splinter E., Grosveld F., De L. W. (2002) Looping and interaction between hypersensitive sites in the active beta-globin locus. Mol Cell 10(6):1453

6. Visel A, Blow MJ, Li Z, Zhang T, Akiyama JA, Holt A, Plajzer-Frick I, Shoukry M, Wright C, Chen F (2009) Chip-seq accurately predicts tissue-specific activity of enhancers. Nature 457(7231):854-8

7. Thurman RE, Rynes E, Humbert R, Vierstra J, Maurano MT, Haugen E, Sheffield NC, Stergachis AB, Wang H, Vernot B (2012) The accessible chromatin landscape of the human genome. Nature 489(7414):75-82

8. Consortium TEP (2012) An integrated encyclopedia of dna elements in the human genome. Nature 489(7414):57-74

9. Johnson DS, Mortazavi A, Myers RM, Wold B (2007) Genome-wide mapping of in vivo protein-dna interactions. Science 316(5830):1497-502

10. Barski A, Cuddapah S, Cui K, Roh TY, Schones DE, Wang Z, Wei G, Chepelev I, Zhao K (2007) High-resolution profiling of histone methylations in the human genome. Cell 129(4):823

11. Hnisz D, Abraham BJ, Lee TI, Lau A, Saintandre V, Sigova AA, Hoke HA Young RA (2013) Super-enhancers in the control of cell identity and disease. Cell 155(4):934

12. Whyte W, Orlando D, Hnisz D, Abraham B, Lin C, Kagey M, Rahl P, Lee TI, Young R (2013) Master transcription factors and mediator establish super-enhancers at key cell identity genes. Cell 153(2):307-19

13. Loven J, Hoke HA, Lin CY, Lau A, Orlando DA, Vakoc CR, Bradner JE, Tong IL, Young RA (2013) Selective inhibition of tumor oncogenes by disruption of super-enhancers. Cell 153(2):320-34

14. Vahedi G, Kanno Y, Furumoto Y, Jiang K, Parker SC, Erdos M, Davis SR, Roychoudhuri R, Restifo NP, Gadina M (2015) Stretch-enhancers delineate disease-associated regulatory nodes in t cells. Nature 520(7548):558-62

15. Witte S, Bradley A, Enright AJ, Muljo SA (2015) High-density p300 enhancers control cell state transitions. Bmc Genomics 16(1):903

16. Khan A, Zhang X (2017) Analysis and prediction of super-enhancers using sequence and chromatin signatures[J]. bioRxiv. 105262. https://doi.org/ 10.1101/105262. https://doi.org/10.1038/s41598-019-38979-9

17. Alipanahi B, Delong A, Weirauch MT, Frey BJ (2015) Predicting the sequence specificities of dna- and rna-binding proteins by deep learning. Nat Biotechnol 33(8):831

18. Zhou J, Troyanskaya OG (2015) Predicting effects of noncoding variants with deep learning" cbased sequence model. Nat Methods 12(10):931

19. Rumelhart DE, Hinton GE, Williams RJ (1986) Learning representations by back-propagating errors. Nature 323(6088):533

20. Langmead B, Trapnell C, Pop M., Salzberg SL (2009) Ultrafast and memory-efficient alignment of short dna sequences to the human genome. Genome Biology 10(3):25
21. Srivastava N, Hinton G, Krizhevsky A, Sutskever I, Salakhutdinov R (2014) Dropout: a simple way to prevent neural networks from overfitting. J Mach Learn Res 15(1):1929-58

22. Kingma DP, Ba J (2014) Adam: A method for stochastic optimization. arXiv preprint arXiv:1412.6980

23. Abadi M, Agarwal A, Barham P, Brevdo E, Chen Z, Citro C, Corrado GS, Davis A, Dean J, Devin M, Ghemawat S, Goodfellow I, Harp A, Irving G, Isard M, Jia Y, Jozefowicz R, Kaiser L, Kudlur M, Levenberg J, Mané D, Monga R, Moore S, Murray D, Olah C, Schuster M, Shlens J, Steiner B, Sutskever I, Talwar K, Tucker $P$, Vanhoucke $V$, Vasudevan V, Viégas F, Vinyals $O$, Warden P, Wattenberg M, Wicke M, Yu Y, Zheng X (2015) TensorFlow: Large-Scale Machine Learning on Heterogeneous Systems. Software available from tensorflow.org http://tensorflow.org/. Accessed 1 Oct 2017

\section{Publisher's Note}

Springer Nature remains neutral with regard to jurisdictional claims in published maps and institutional affiliations.
Ready to submit your research? Choose BMC and benefit from:

- fast, convenient online submission

- thorough peer review by experienced researchers in your field

- rapid publication on acceptance

- support for research data, including large and complex data types

- gold Open Access which fosters wider collaboration and increased citations

- maximum visibility for your research: over $100 \mathrm{M}$ website views per year

At BMC, research is always in progress.

Learn more biomedcentral.com/submissions 